\title{
LZTR1 facilitates polyubiquitination and degradation of RAS-GTPases
}

\author{
Taiki Abe $\mathbb{I}^{1} \cdot$ Ikumi Umeki $^{1} \cdot$ Shin-ichiro Kanno ${ }^{2} \cdot$ Shin-ichi Inoue ${ }^{1} \cdot$ Tetsuya Niihori $^{1} \cdot$ Yoko Aoki $^{1}$
}

Received: 7 February 2019 / Revised: 4 July 2019 / Accepted: 8 July 2019 / Published online: 23 July 2019

(c) The Author(s) 2019

\begin{abstract}
Leucine zipper-like transcriptional regulator 1 (LZTR1) encodes a member of the BTB-Kelch superfamily, which interacts with the Cullin3 (CUL3)-based E3 ubiquitin ligase complex. Mutations in LZTR1 have been identified in glioblastoma, schwannomatosis, and Noonan syndrome. However, the functional role of LZTR1 in carcinogenesis or human development is not fully understood. Here, we demonstrate that LZTR1 facilitates the polyubiquitination and degradation of RAS via the ubiquitin-proteasome pathway, leading to the inhibition of the RAS/MAPK signaling. The polyubiquitination and degradation of RAS was also observed in cells expressing MRAS, HRAS, NRAS, and KRAS as well as oncogenic RAS mutants and inhibited the activation of ERK1/2 and cell growth. In vivo ubiquitination assays showed that MRAS-K127 and HRAS-K170 were ubiquitinated by LZTR1 and that the polyubiquitinated-chains contained mainly Ub-K48, K63, and K33linked chains, suggesting its possible involvement in autophagy. Immunoprecipitation analyses showed the interaction of LZTR1 and RAS-GTPases with autophagy-related proteins, including LC3B and SQSTM1/p62. Co-expression of LZTR1 and RAS increased the expression of lipidated form of LC3B. However, long-term treatment with chloroquine had little effect on RAS protein levels, suggesting that the contribution of autophagy to LZTR1-mediated RAS degradation is minimal. Taken together, these results show that LZTR1 functions as a "RAS killer protein" mainly via the ubiquitinproteasome pathway regardless of the type of RAS GTPase, controlling downstream signal transduction. Our results also suggest a possible association of LZTR1 and RAS-GTPases with the autophagy. These findings provide clues for the elucidation of the mechanisms of RAS degradation and regulation of the RAS/MAPK signaling cascade.
\end{abstract}

\section{Introduction}

LZTR1 encodes leucine zipper-like transcription regulator 1 (LZTR1), which is a Golgi protein and that belongs to the

Edited by V. D'Angiolella

Supplementary information The online version of this article (https:// doi.org/10.1038/s41418-019-0395-5) contains supplementary material, which is available to authorized users.

Taiki Abe

abet@med.tohoku.ac.jp

$\checkmark$ Yoko Aoki

aokiy@med.tohoku.ac.jp

1 Department of Medical Genetics, Tohoku University School of Medicine, Seiryo-machi 1-1, Aobaku, Sendai 980-8574, Japan

2 Division of Dynamic Proteome, Institute of Development, Aging, and Cancer, Tohoku University, Seiryo-machi 4-1, Aobaku, Sendai 980-8575, Japan
BTB-Kelch superfamily [1, 2]. LZTR1 has a unique structure compared to other BTB-Kelch family members. Typical BTB-Kelch proteins contain an N-terminal BTB domain followed by one Kelch motif. In contrast, LZTR1 contains an N-terminal Kelch motif followed by two BTB domains. In general, members of the BTB-Kelch superfamily function as substrate adaptor proteins for Cullin3 (CUL3)-based E3 ubiquitin ligases [3, 4]. It has been reported that LZTR1 might be involved in apoptosis and ubiquitination, but the substrate molecules of LZTR1 had not been identified $[1,5-7]$.

LZTRI is a known tumor suppressor gene, and its germline and somatic mutations have been identified in patients with schwannomatosis and glioblastoma, which is one of the most challenging cancers to treat $[2,7,8]$. Additionally, LZTRI has been described as a causative gene of Noonan syndrome $[9,10]$. Noonan syndrome is a relatively common autosomal dominant or recessive inherited disorder, that is characterized by congenital heart disease, mental retardation, and increased cancer risk [11]. Noonan 
syndrome and related disorders have been called RASopathies, which are caused by germline mutations in genes encoding various components of the RAS/MAPK signaling pathway, including PTPN11, HRAS, KRAS, NRAS, RRAS, BRAF, RAF1, SOS1, SHOC2, and CBL [12, 13]. Recent advances in next-generation sequencing (NGS) have identified additional genes involved in RASopathies, including RIT1, A2ML1, LZTR1, SOS2, PPPICB, and $M R A S[9,14-17]$. However, the functional roles of recently identified genes are not fully understood. Indeed, no evidence regarding the functional interaction between LZTR1 and the RAS/MAPK signaling pathway had been reported.

Two observations have emerged indicating that LZTR1 is potentially associated with the RAS/MAPK signaling pathway. We have reported that LZTR1 interacts with the RAF1/SHOC2/PPP1CB complex and promotes RAF1Ser259 phosphorylation, leading to the inactivation of the MAPK signaling pathway [18]. Another observation was reported by two research groups, who found that LZTR1 promotes the site-specific mono- and di-ubiquitination of RAS and attenuates the association of ubiquitinated-RAS with the plasma membrane $[19,20]$. However, it is not fully understood whether LZTR1 functionally interacts with RAS family proteins. Based on these findings, we examined whether LZTR1 functionally interacts with RAS proteins and controls the activities of downstream signals, including the MAPK signaling pathway.

\section{Materials and Methods}

\section{Cell culture}

HEK293 cells from ATCC (Manassas, VA, USA) were cultured in Dulbecco's Modified Eagle's Medium (DMEM, Thermo Fisher Scientific, Waltham, MA, USA) supplemented with $10 \%$ fetal bovine serum (FBS) and Antibiotic-Antimycotic solution (Thermo Fisher Scientific) at $37{ }^{\circ} \mathrm{C}$ in a $5 \% \mathrm{CO}_{2}$ atmosphere. Lipofectamine 3000 (Thermo Fisher Scientific) was used for transient transfection of plasmids according to the manufacturer's instructions.

\section{Target gene expression plasmids}

The Flag-KRAS-pCAGGS plasmid has been described previously [21]. cDNAs encoding LZTR1, MRAS, KRAS, HRAS, NRAS, ubiquitin (Ub), and CUL3 were amplified by PCR with specific primer sets from human cDNA or the Flag-KRAS-pCAGGS plasmid (Table S1), and they were inserted into the pENTR-SD-D-TOPO vector (Thermo Fisher Scientific) using KOD-Plus-Neo (Toyobo, Osaka, Japan). Epitope tags (Flag, Myc, and HA) and single base substitutions were inserted and mutated using specific primers and the KOD-Plus-Mutagenesis Kit (Toyobo), respectively (Supplementary Table S1). pENTR-SD-DTOPO constructs were subcloned into the pcDNA3.2/V5DEST and pLenti6.3/TO/V5-DEST vectors (Thermo Fisher Scientific) using the Gateway cloning system.

\section{Small interfering RNA transfection}

HEK293 cells were transfected with $10 \mathrm{nM}$ ONTARGETplus Non-Targeting Pool (control-siRNA) or 10 nM ON-TARGETplus SMART Pool-human LZTR1 (LZTR1 siRNA) (GE Healthcare Dharmacon, Lafayette, CO) using Lipofectamine RNAiMAX (Thermo Fisher Scientific) following the manufacturer's protocol.

\section{Cycloheximide-chase assays}

HEK293 cells were transfected with the indicated expression plasmids for $48 \mathrm{~h}$, and then the cells were treated with $50 \mu \mathrm{g} / \mathrm{mL}$ cycloheximide (CHX). Chronological changes in Flag-RAS protein levels were evaluated by western blot analyses.

\section{Generation of the LZTR1 knockout cell line using the CRISPR/Cas9 system}

We designed a gRNA to target the first exon of LZTRI utilizing CHOPCHOP (http://chopchop.cbu.uib.no/index.php) and generated the gRNA using specific primers (Table S1) and a GeneArt Precision gRNA Synthesis kit (Thermo Fisher Scientific) following the manufacturer's protocol. HEK293 cells were transfected with the gRNA and Cas9 nuclease protein NLS (Nippon Gene, Tokyo, Japan) using Lipofectamine CRISPRMAX Transfection Reagent (Thermo Fisher Scientific). Seventy-two hours later, the cleavage efficiency of LZTR1-targeting gRNA/Cas9 was evaluated, and then the cells were reseeded at 0.7 cells per well in 96-well culture plates to isolate LZTRI knockout clones. Two weeks after reseeding, we picked up clones and verified the target sequence. The Sanger sequencing method has been described previously [18]. The LZTRI-knockout cell line was named 293LZTRI KO.

\section{Construction of recombinant lentiviruses and generation of stable cell lines}

The open reading frame of Myc-tagged LZTR1 was subcloned into the pLenti6.3/TO/V5-DEST plasmid according to the ViraPower HiPerform T-Rex Gateway Expression System manufacturer's protocols (Thermo Fisher Scientific). The day before transfection, 293FT cells (Thermo 
Fisher Scientific) were seeded at $5 \times 10^{6}$ cells $/ 10 \mathrm{~cm}$ dish and then transfected with pLenti-6.3/TO-Myc-LZTR1 or pLenti6.3/TO-LacZ-V5. The next day, we removed and discarded the medium and replaced it with complete culture medium. Seventy-two hours after transfection, we harvested and centrifuged the lentivirus-containing supernatants. We stored the centrifuged supernatants as lentiviral stocks at $-80{ }^{\circ} \mathrm{C}$.We infected HEK293 cells with lentiviruses containing the gene of interest (LacZ-V5 or Myc-LZTR1) for $24 \mathrm{~h}$, and then positive cells were selected by blasticidin (Thermo Fisher Scientific). Approximately 2 weeks later, we seeded these cells and evaluated cell growth.

\section{Co-immunoprecipitation assays}

HEK293 cells were transfected with expression plasmids using Lipofectamine 3000 reagent (Thermo Fisher Scientific). Forty-eight hours later, the cells were treated with $10 \mu \mathrm{M}$ MG132 for $6 \mathrm{~h}$ and then lysed using cell lysis/ binding buffer $(50 \mathrm{mM}$ Tris $\mathrm{HCl} \mathrm{pH}$ 7.4, $150 \mathrm{mM} \mathrm{NaCl}, 1$ mM EDTA, and 1\% Triton X-100). The cell extracts were rotated with Flag M2 affinity gel (Sigma-Aldrich, St. Louis, MO, USA) or anti-Ras Magnetic Bead Conjugate Antibody (Millipore) at $4{ }^{\circ} \mathrm{C}$. After washing the gels three times with Tris-based wash buffer and once with PBS, the immune complexes were eluted with $3 \times$ Flag-peptide and analyzed by western blot.

\section{Western blot analyses}

Whole cell lysates were prepared by using CelLytic MT Cell Lysis Reagent (Sigma Aldrich), and the cell lysates were centrifuged at $15,000 \times g$ for $15 \mathrm{~min}$ at $4{ }^{\circ} \mathrm{C}$. The protein concentration of the supernatant was determined using the Bio-Rad Protein Assay (Bio-Rad Laboratories, Hercules, CA). The lysates were subjected to SDS-PAGE (4-20\% Criterion TGX Precast Gels; Bio-Rad Laboratories) and transferred to PVDF membranes (Bio-Rad Laboratories). After blocking with 5\% nonfat milk, the membranes were incubated overnight at $4{ }^{\circ} \mathrm{C}$ with the following antibodies (the catalog numbers are in parentheses). The antibodies (with catalog numbers in parentheses): ERK1/2 (9102), phospho-ERK1/2 (9101), Myc-tag (2278), HA-tag (3724), K48-linkage Specific Polyubiquitin (4289), K63-linkage Specific Polyubiquitin (D7A11) Rabbit mAb (5621), SQSTM1/p62 (5114), and LC3B (3868) were purchased from Cell Signaling (Danvers, MA, USA). Flag-tag (F1804), Monoclonal Anti-Flag M2 peroxidase (HRP) antibody (A8592) and $\beta$-actin (A5316) were from Sigma Aldrich. LZTR1 (sc-390166), pan-RAS (05-516), RAS (ab52939) and V5-tag (M215-3) antibodies were from Santa Cruz Biotechnology (Santa Cruz, CA, USA), Millipore (Bedford, MA),
Abcam (Cambridge, UK) and MBL (Nagoya, Japan), respectively.

\section{Immunofluorescence analyses}

HEK293 cells grown on $13 \mathrm{~mm}^{2}$ glass coverslips (Matsunami Glass, Osaka, Japan) were transfected with expression plasmids. Forty-eight hours later, the cells were fixed in $4 \%$ paraformaldehyde for $15 \mathrm{~min}$ and rinsed three times with PBS. After washing, the fixed cells were incubated in PBS containing $0.1 \%$ Triton $\mathrm{X}-100$ for $10 \mathrm{~min}$, rinsed three times with PBS and incubated in BlockAid Blocking solution (Thermo Fisher Scientific) for $1 \mathrm{~h}$ at room temperature. The coverslips were incubated overnight at $4{ }^{\circ} \mathrm{C}$ with primary antibodies diluted in Can Get Signal Immunostain Immunoreaction Enhancer Solution (Toyobo), washed with PBS/ $0.1 \%$ Tween-20, and further incubated with secondary antibodies for $1 \mathrm{~h}$ at room temperature. Finally, the coverslips were examined by confocal fluorescence microscopy.

\section{In vivo ubiquitination assays}

HEK293 cells were transfected with the indicated plasmids and treated with $10 \mu \mathrm{M}$ MG132 (Sigma Aldrich) for 4-6 h before harvest. The cells were lysed in hot lysis buffer containing 1\% SDS and $10 \mathrm{mM}$ N-ethylmaleimide (NEM, Sigma Aldrich). The lysates were boiled at $100{ }^{\circ} \mathrm{C}$ for 10 min to disrupt noncovalent protein-protein interactions and were diluted 1:10 with SDS-free cell lysis buffer to reduce the concentration of SDS. The diluted lysates were immunoprecipitated with anti-Flag-M2 agarose and evaluated by western blot analyses.

\section{Quantitative reverse transcription-PCR (qRT-PCR)}

Total RNA preparation and cDNA syntheses were carried out using TRIzol and a High-Capacity cDNA Reverse Transcription Kit (Thermo Fisher Scientific), respectively. qRT-PCR was carried out using FastStart Universal Probe Master (ROX) (Applied Biosystems, Foster City, CA) with StepOnePlus (Applied Biosystems). Amplification primers and hydrolysis probes were designed using the Universal ProbeLibrary Assay Design Center (https://qpcr. probefinder.com/roche3.html, and Table S3). The mRNA levels were normalized to GAPDH mRNA levels.

\section{Statistical analyses}

Statistical analyses were performed using JMP Pro 13 software (SAS Institute, Cary, NC). All data are provided as the mean $\pm S D$. Significant differences between the control and treatment groups were assessed by Student's $t$ test. 

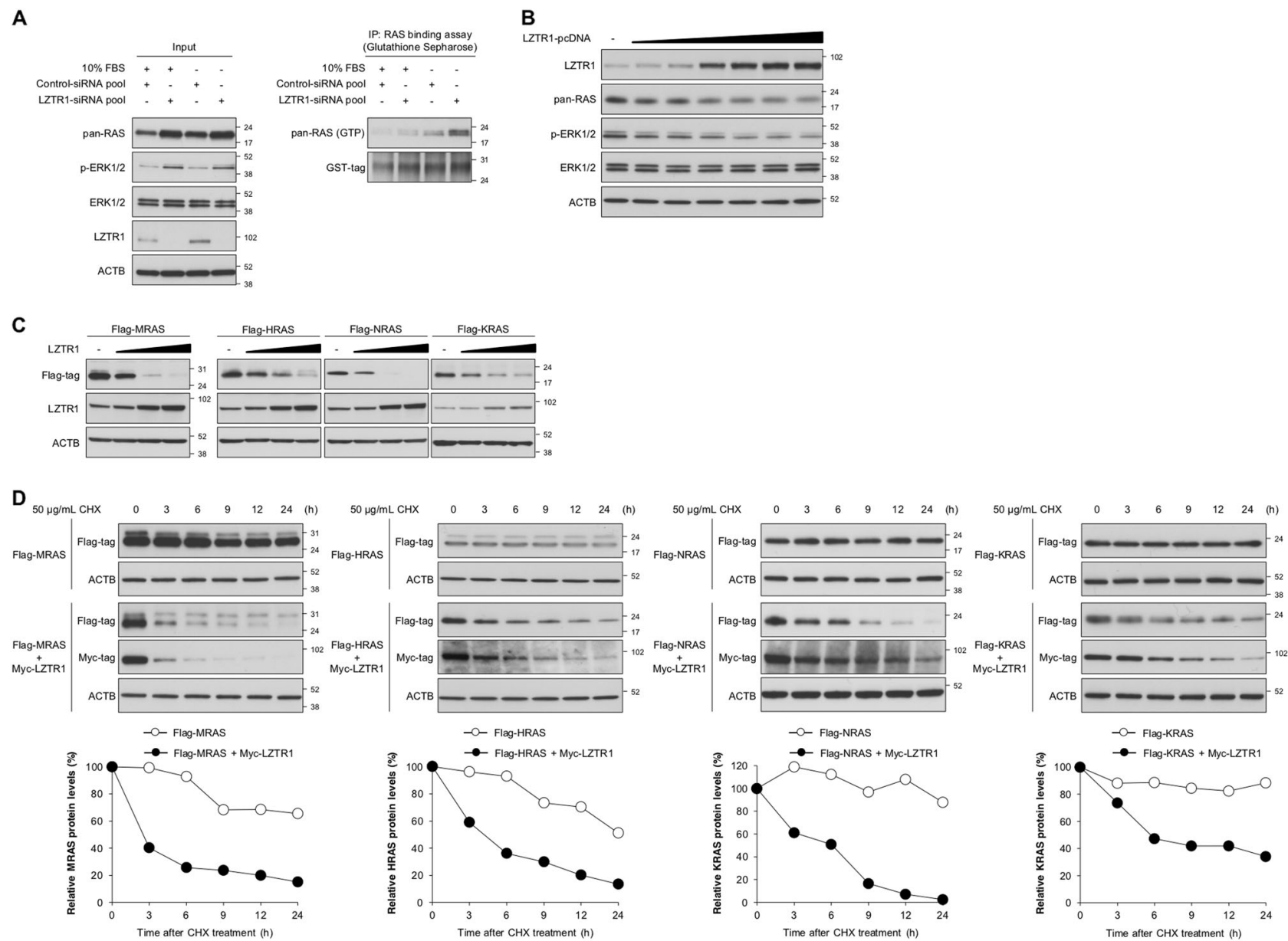

Fig. 1 LZTR1 regulates the expression of four RAS GTPases, including MRAS, HRAS, NRAS, and KRAS. a HEK293 cells were transfected with control-siRNA pool or LZTR1-targeting-siRNA pool (LZTR1-siRNA pool). Twenty-four hours later, cells were cultured in serum-free or $10 \%$ serum-containing medium for an additional $24 \mathrm{~h}$, and we evaluated the expression and activity levels of RAS and the RAS/MAPK signaling pathway by western blot. b, $\mathbf{c}$ The influence of LZTR1 overexpression on endogenous and exogenous RAS protein levels. HEK293 cells were seeded in $60 \mathrm{~mm}$ dishes and transfected with the indicated plasmids for $24 \mathrm{~h}$, and cells were cultured in fresh medium for an additional $24 \mathrm{~h}$. Then we evaluated the protein levels at $48 \mathrm{~h}$ after transfection. b Cells were transfected with $0,0.5,1,2,3,4$,

\section{Results}

\section{LZTR1 controls the expression of four RAS GTPases}

To evaluate the effect of LZTR1 on the RAS/MAPK signaling pathway, we knocked down LZTRI in HEK293 cells using siRNA. The protein levels of phospho-ERK1/2 (pERK1/2) were markedly increased in LZTRl-knockdown cells, but total-ERK1/2 levels were not changed (Fig. 1a left panel). We searched for molecules that were changed by LZTR1-siRNA and found that the protein levels of pan-RAS (HRAS, NRAS, and KRAS) were increased (Fig. 1a left panel). The expression levels of activated-RAS (GTP-RAS)

5, $6 \mu \mathrm{g}$ LZTR1-pcDNA only. (c) Cells were transfected with Flaggtagged RAS and LZTR1 $(2.5: 0 \mu \mathrm{g}, 2.5: 0.5 \mu \mathrm{g}, 2.5: 1 \mu \mathrm{g}$ or 2.5: $2.5 \mu \mathrm{g}$ ratios of RAS:LZTR1). d To perform the cycloheximide (CHX)-chase assay, each $2.5 \mu \mathrm{g}$ Flag-RAS- and $1 \mu \mathrm{g}$ Myc-LZTR1-expressing plasmid or empty plasmid was transfected into HEK293 cells for $48 \mathrm{~h}$; then, cells were treated with $50 \mu \mathrm{g} / \mathrm{mL}$ cycloheximide (CHX). The cells were harvested at the indicated time points after treatment, and Flag-RAS expression levels were evaluated by western blot analyses. Band densities were analyzed using ImageJ software from the National Institutes of Health, and expression was normalized to ACTB protein levels

were also increased in immunoprecipitants with the agaroseconjugated RAF1/RAS-binding domain (RBD) (Fig. 1a, right panel). Endogenous RAS was also increased in LZTRI knockout cells generated by the CRISPR/Cas9 system (293LZTRI KO cells, Supplementary Fig. S1a). In contrast, the overexpression of LZTR1 decreased the protein levels of panRAS and p-ERK1/2 in a dose-dependent manner (Fig. 1b). Pan-RAS and p-ERK1/2 protein levels and cell growth were decreased in cells stably expressing Myc-LZTR1 (Supplementary Fig. S1b). These results suggest that LZTR1 regulates endogenous RAS expression, and the LZTR1-mediated downregulation of endogenous RAS, at least in part, causes cell growth inhibition. 
Next, we investigated whether LZTR1 regulates the expression of various types of RAS GTPases. The expression of four Flag-RAS proteins, including MRAS, HRAS, NRAS, and KRAS, drastically decreased with LZTR1 co-expression in a dose-dependent manner (Fig. 1c). This phenomenon was also observed even when RAS and LZTR1 were individually transfected into HEK293 cells at an interval of $24 \mathrm{~h}$ (Supplementary Fig. S1c). This LZTR1-dependent RAS downregulation was detected in cells expressing oncogenic mutants of RAS-expressing cells, including MRAS-G22V, HRASG12V, NRAS-G12V, and KRAS-G12V, but it seemed to be weak compared with wild-type RAS-GTPases (Supplementary Fig. S1d).

Subsequently, we carried out cycloheximide (CHX)chase assays and measured the stability of RAS proteins. In cells expressing Flag-MRAS alone, Flag-MRAS gradually decreased up to $24 \mathrm{~h}$ after treatment with CHX (Fig. 1d, left panel). In contrast, in cells co-expressing Flag-MRAS and Myc-LZTR1, Flag-MRAS expression was markedly decreased under the same conditions (Fig. 1d, left panel). A drastic decrease in RAS expression was also observed in Myc-LZTR1 and Flag-HRAS or Flag-KRAS co-expressing cells (Fig. 1d, center and right panel). LZTR1 overexpression did not significantly alter the mRNA levels of the endogenous RAS family members (Supplementary Fig. S1e). These results indicate that LZTR1 controls the expression of RAS proteins by promoting their degradation.

\section{LZTR1 promotes the polyubiquitination and degradation of four RAS proteins}

Since LZTR1 is a substrate adaptor of CUL3-based ubiquitin E3 ligase, we examined whether the inhibition of the ubiquitin-proteasome pathway rescues LZTR1mediated RAS degradation. LZTR1-dependent proteolysis of RAS was recovered by treatment with a proteasome inhibitor, MG132 (Fig. 2a and Supplementary Fig. S2a). In in vivo ubiquitination assays using HA-tagged wild-type ubiquitin (HA-Ub), LZTR1 co-expression drastically increased the polyubiquitination of endogenous RAS-GTPases with or without MG132, but a more significant increase was observed in MG132-treated cells. LZTR1-mediated ubiquitin chains on RAS included UbK48- and Ub-K63-linkage specific polyubiquitin chains (Fig. 2b). The enhancement of RAS polyubiquitination by LZTR 1 was also detected in cells expressing exogenous Flag-RAS-GTPases, including MRAS, HRAS, NRAS, and KRAS (Fig. 2c). Moreover, LZTR1-mediated RAS polyubiquitination was observed in cells expressing oncogenic mutants of RAS (MRAS-G22V and HRASG12V) (Supplementary Fig. S2b). These data demonstrated that LZTR1 facilitates the polyubiquitination of four RAS proteins, leading to their degradation, at least in part.

Next, we examined how LZTR1 functionally interacts with RAS proteins in LZTR1-mediated RAS polyubiquitination. Immunofluorescence analysis showed that RAS proteins were present in the cytoplasm and around the plasma membrane of HEK293 cells expressing Flag-RAS alone, whereas Flag-RAS proteins showed a dotted pattern and colocalized in cells co-expressing Flag-RAS and MycLZTR1 (Fig. 3a and Supplementary Fig. S3). Coimmunoprecipitation assays demonstrated that RAS could interact with LZTR1 and CUL3 (Fig. 3b). LZTR1 has one Kelch and two BTB domains (Fig. 3c). For instance, KEAP1, one of the BTB-Kelch superfamily members, interacts with CUL3 through its BTB domain and captures NRF2 through its Kelch domain as a substrate adaptor for the CUL3-based E3 complex [22]. Co-immunoprecipitation assays showed that RAS interacted with the LZTR1-Kelch and/or BTB1 domain and that Flag-CUL3 strongly bound to the LZTR1-BTB1 domain and weakly interacted with the LZTR1-Kelch domain (Fig. 3d, e). BTB family proteins are able to homo or heterodimerize with themselves or other BTB proteins, and this BTB-BTB dimerization could affect signal transduction [23-26]. As expected, Myc-LZTR1 dimerized with Flag-LZTR1 (Fig. 3f). These results suggest that LZTR1 homodimerizes with itself and captures RAS proteins as a substrate adaptor for CUL3-based E3 ligase (Fig. 3g).

\section{The analysis of target Lys residues of RAS ubiquitinated by LZTR1}

To uncover the molecular mechanism underlying LZTR1-mediated RAS polyubiquitination, we examined which Lys residues are target residues in overexpressed LZTR1-dependent RAS polyubiquitination among six highly conserved Lys residues (K5/15, K42/52, K88/97, K104/114, K117/127, K147/158) in MRAS, HRAS, NRAS and KRAS (Fig. 4a). We generated MRAS-1KR mutants, in which only one of the six Lys residues was mutated to Arg. In vivo ubiquitination assays showed that LZTR1-dependent polyubiquitination was slightly decreased in MRAS-K127R-expressing cells, but K127R mutation did not attenuate MRAS degradation (Fig. 4b).

To further investigate whether MRAS-K127 is one of the target residues in overexpressed LZTR1-mediated polyubiquitination, we constructed the MRAS-6KR mutant, in which six Lys residues of RAS were mutated to Arg, and the MRAS-6KR-based mutant (MRAS-5KR), in which only one of the six Lys residues remained. The expression level of Flag-MRAS-5KR-K127 was unexpectedly higher 
A
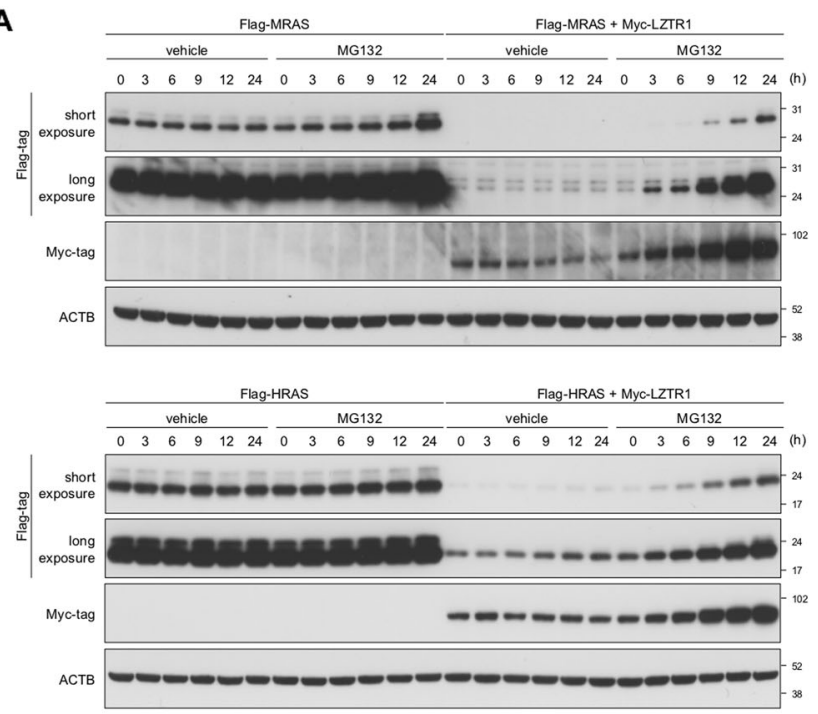

C

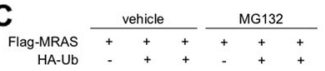
MYcLATE
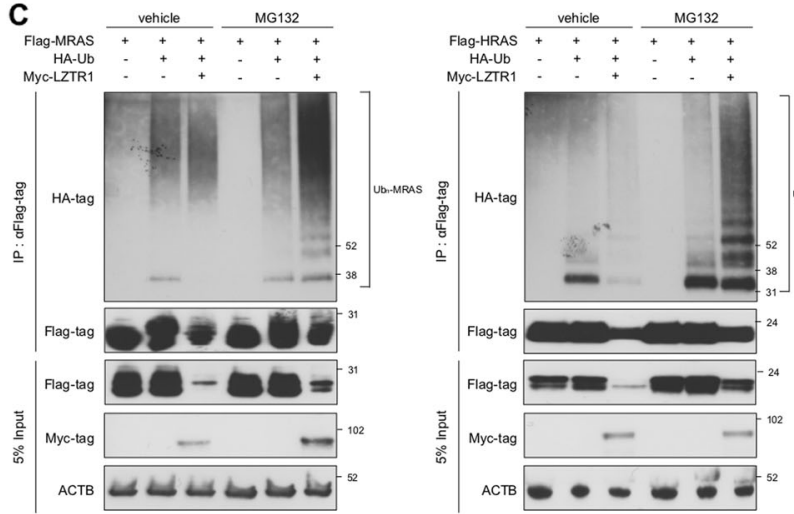

Fig. 2 LZTR1 promotes the polyubiquitination of four RAS GTPases (MRAS, HRAS, NRAS, and KRAS), leading to their degradation. a HEK293 cells were transfected with $2.5 \mu \mathrm{g}$ MRAS or HRASexpressing plasmid in the presence or absence of $2.5 \mu \mathrm{g}$ MycLZTR1-expressing plasmid for $48 \mathrm{~h}$ followed by treatment with vehicle $(0.1 \%$ DMSO) or $10 \mu \mathrm{M}$ MG132, a proteasome inhibitor. We then evaluated the chronological changes in Flag-MRAS expression levels by western blot. b HEK293 cells were transfected with HA-Ub $(7 \mu \mathrm{g})$, Myc-LZTR1 $(2 \mu \mathrm{g})$ for $24 \mathrm{~h}$ followed by treatment with vehicle

than that of the other mutants and was comparable to that of wild-type MRAS. MRAS-WT or MRAS-5KR-K127 expression was drastically decreased by LZTR1 co-expression (Supplementary Fig. S4a). The polyubiquitination status increased in cells expressing MRAS5KR-K127, but it was not comparable to that in cells expressing wild-type MRAS (Supplementary Fig. S4b). These data suggest that MRAS-K127 is one of the candidate Lys residues in overexpressed LZTR1-mediated polyubiquitination, but the contribution of K127 ubiquitination may be partial. Furthermore, LZTR1-dependent polyubiquitination was not completely diminished in even MRAS-6KR-expressing cells, suggesting the existence of a ubiquitination target residue other than the six Lys sites we examined.
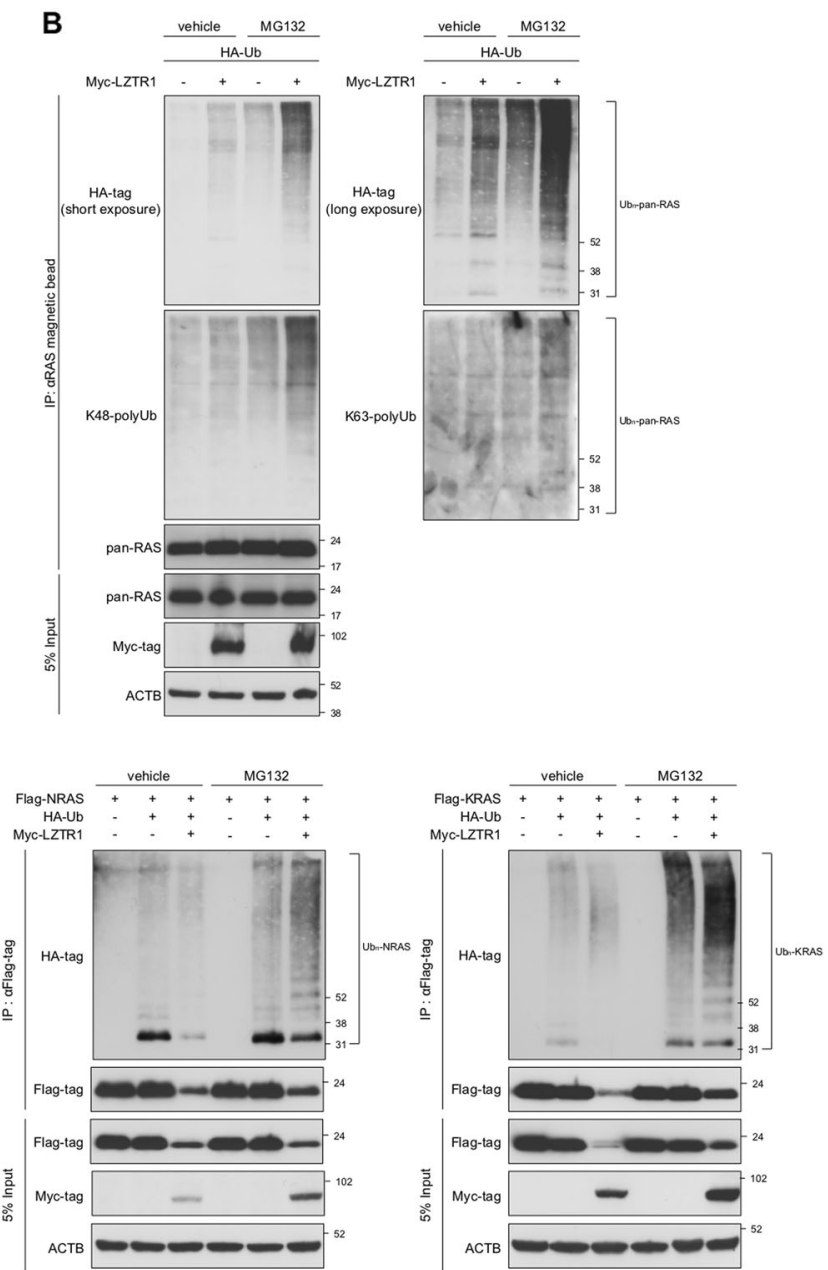

(0.1\% DMSO) or $10 \mu \mathrm{M}$ MG132 for $6 \mathrm{~h}$. Thirty hours after transfection, the cell extracts were subjected to in vivo ubiquitination assays, and we evaluated the ubiquitination status of endogenous RAS. c HEK293 cells were transfected with HA-Ub $(7 \mu \mathrm{g})$, LZTR1 $(2 \mu \mathrm{g})$, and each RAS-expressing plasmid $(10 \mu \mathrm{g})$ for $24 \mathrm{~h}$ followed by treatment with vehicle ( $0.1 \%$ DMSO) or $10 \mu \mathrm{M}$ MG132 for $6 \mathrm{~h}$. Thirty hours later, the cell extracts were subjected to in vivo ubiquitination assays. In this figure, we used MRAS-pcDNA, HRAS-pcDNA, NRAS-pcDNA and KRAS-pCAGGS expression plasmids

A recent study showed that Lys-170 of HRAS is ubiquitinated by LZTR1 [19]. The Lys residue is located in the hypervariable region (HVR), in which less than $10-15 \%$ of residues are identical between RAS proteins. Therefore, K170 was not included in the six common residues we selected in Fig. 4a. The ubiquitination status of the HRASK170R mutant was lower than that of HRAS-WT (Fig. 4c), but HRAS degradation was not attenuated (Fig. 4d). pERK1/2 levels were decreased by LZTR 1 co-expression, but not changed in cells expressing HRAS-K170R or HRAS-G12V/K170R alone (Fig. 4d). These results suggest that Lys-170 in HRAS is one of the target residues of LZTR1-dependent RAS ubiquitination, but the decreased polyubiquitination is not enough for attenuation of RAS degradation. 
A
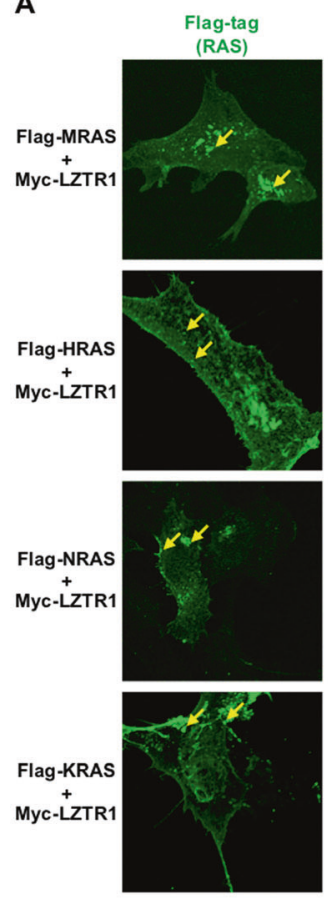
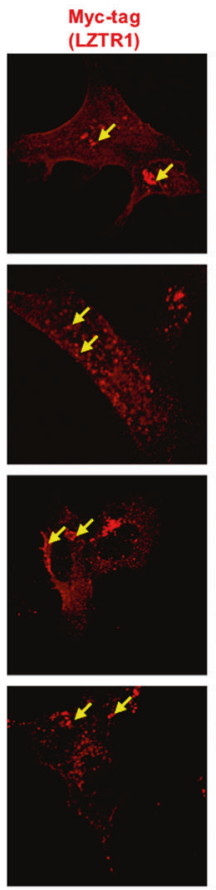

Nuclei
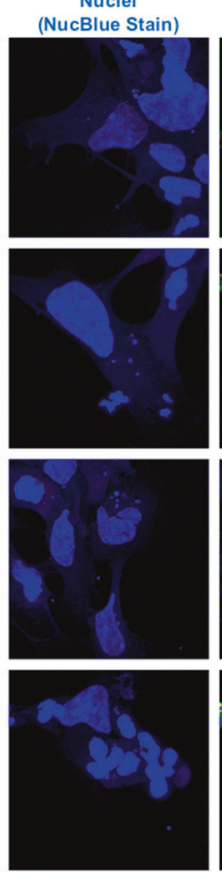
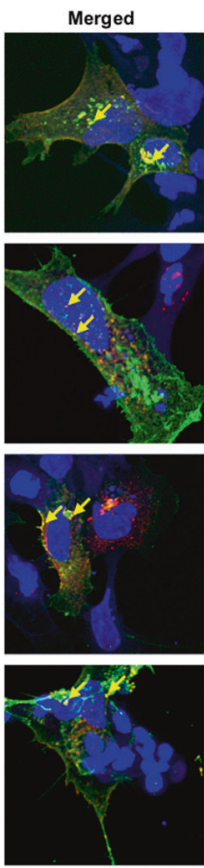

B

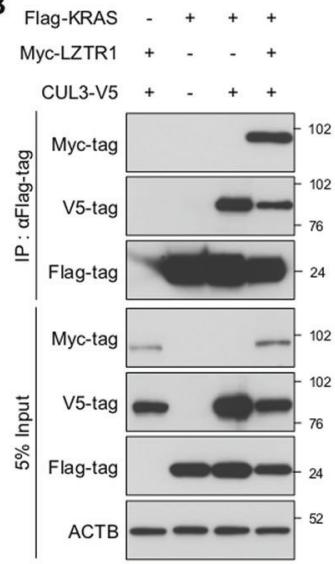

C LZTR1 (90 kDa, Kelch-BTB-BTB protein)

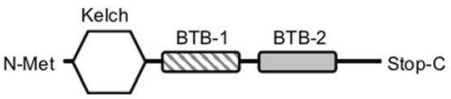

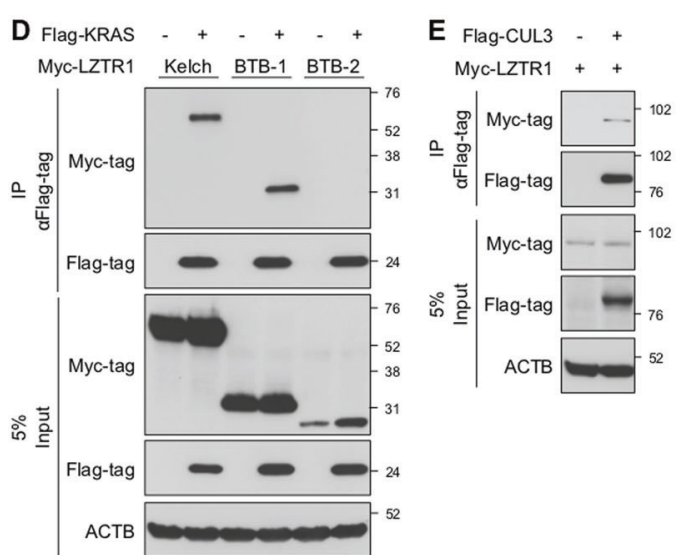
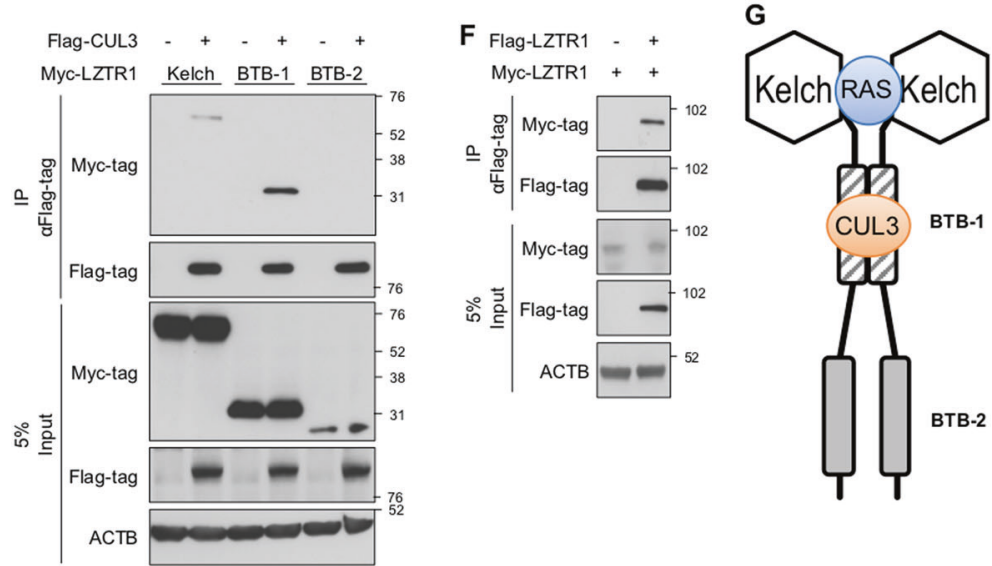

Fig. 3 LZTR1 homodimerizes with itself and forms a complex with RAS and CUL3. a Cellular localization of Myc-LZTR1 and Flag-RAS in HEK293 cells. The cells were transfected with the indicated expression plasmids and stained with anti-Myc-tag (red), anti-Flag-tag (green), and NucBlue Stain (nuclei, blue). Cells were seeded at $5 \times 10^{4}$ cells/well, grown on $13 \mathrm{~mm} 2$ glass coverslips in 24-well plate for $24 \mathrm{~h}$, and then transfected with indicated plasmids. The ratio of RAS and LZTR1 was 5:1 (1 $\mu \mathrm{g}: 0.2 \mu \mathrm{g})$ and we evaluated $30 \mathrm{~h}$ after transfection. The yellow arrows indicate representative locations where LZTR1 and RAS overlap. b Cells were seeded in $100 \mathrm{~mm}$ dishes, transfected with with Flag-KRAS-pCAGGS $(10 \mu \mathrm{g})$, Myc-LZTR1-pcDNA $(4 \mu \mathrm{g})$, and CUL3-V5-pcDNA $(4 \mu \mathrm{g})$ for $30 \mathrm{~h}$. The lysates were subjected to immunoprecipitation assays with Flag-M2 agarose, and then we

\section{LZTR1-mediated RAS polyubiquitination is formed by multiple types of ubiquitin chains}

Seven Lys and N-terminal Met residues of ubiquitin form homogeneous, multiple mixed, or branched chains. Although the functions of the multiple mixed and branched evaluated their interactions by western blot analyses using anti-Flagtag, anti-Myc-tag, anti-V5, and anti- $\beta$ actin antibodies. c A schematic diagram of the domain organization of LZTR1. d-f HEK293 cells were transfected with the indicated expression plasmids, and the lysates were subjected to co-immunoprecipitation assays. The immunoprecipitants were subjected to western blot analyses using anti-Flag-tag, anti-Myc-tag, and anti- $\beta$ actin antibodies. In (d), cells were transfected with $7.5 \mu \mathrm{g}$ Flag-KRAS-pCAGGS and $7.5 \mu \mathrm{g}$ FlagLZTR1 domains for $48 \mathrm{~h}$. In (e), cells were transfected with $7.5 \mu \mathrm{g}$ Flag-CUL3-pcDNA and 7.5 $\mu$ g Myc-LZTR1-domain-pcDNAs for 48 h. In (f), cells were transfected with $7.5 \mu \mathrm{g}$ Flag-LZTR1-pcDNA and $7.5 \mu \mathrm{g}$ Myc-LZTR1-pcDNAs for $48 \mathrm{~h}$. $\mathrm{g}$ A schematic diagram of the homodimerized-LZTR1/RAS/CUL3 complex

chains remain unclear, those of typical homogenous Lyslinked ubiquitin chains are becoming apparent [27, 28]. For instance, K48-linkage-specific polyubiquitination promotes proteasome-mediated proteolysis, while K63-linkagespecific polyubiquitination regulates numerous functions of target proteins such as protein-protein interactions, 

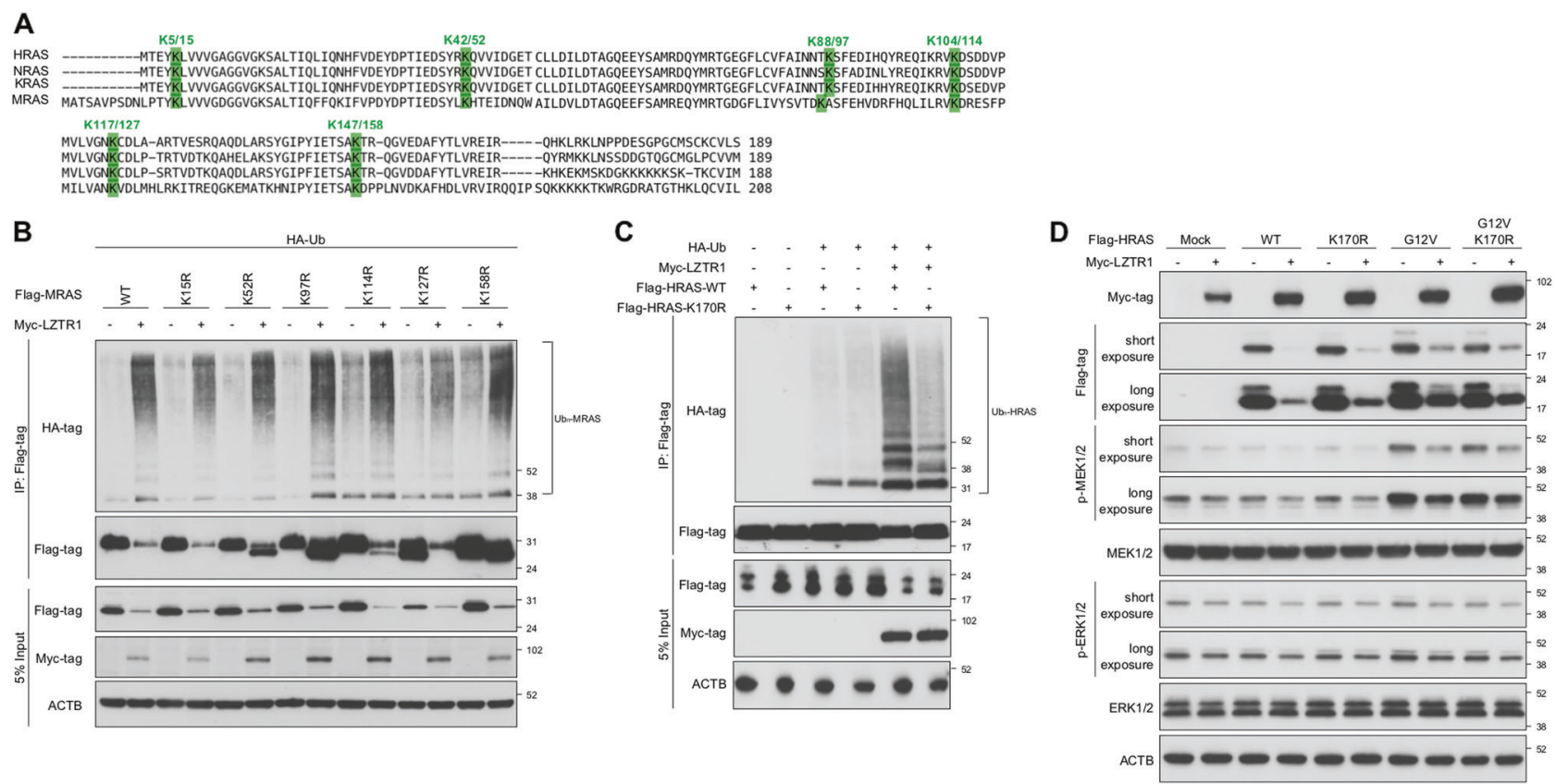

Fig. 4 The analysis of target Lys residues of RAS ubiquitinated by LZTR1. a A schematic diagram of the sequence alignments of HRAS, NRAS, KRAS, and MRAS. b In vivo ubiquitination assays were performed with MRAS-1KR mutants instead of wild-type. HEK293 cells were transfected with the indicated plasmids for $24 \mathrm{~h}$ followed by treatment with MG132 for $6 \mathrm{~h}$. Thirty hours later, the polyubiquitination status was evaluated by western blot using anti-HA-tag, anti-Flag-tag, anti-Myc-tag, and anti- $\beta$ actin antibodies. In (b), cells were seeded in $100 \mathrm{~mm}$ dishes and transfected with transfected with

intracellular localization, DNA repair, endocytosis, and transport [29].

In Fig. 2b, Ub-K48- and Ub-K63-linkage specific polyubiquitin chains were present in the LZTR1-mediated polyubiquitin chains on RAS. Then, we generated eight constructs expressing ubiquitin mutants, including Ub-K0, in which all seven Lys residues were mutated to Arg to prevent chain formation and polyubiquitination. The other mutants were $\mathrm{HA}-\mathrm{Ub}-\mathrm{K} 0$-based mutants, in which only one of the seven Lys residues remained. The RAS polyubiquitination status decreased in cells expressing HAUb-K0 (Fig. 5a). Some ubiquitin mutants promoted the polyubiquitination of RAS. Of these mutants, the weakest suppression of polyubiquitination was observed in Ub-K33 mutant-expressing cells (Fig. 5a). These results demonstrate that LZTR1-mediated RAS polyubiquitination involves not only a particular Lys residue-based chain but also multiple Lys residue-based chains, including mixed or branched chains.

To further examine the contributions of typical (K48and K63-linked chains) and atypical ubiquitination (K33linked chain) to LZTR1-mediated RAS polyubiquitination, in vivo ubiquitination assays were carried out with Ub-1KR mutants, in which only one of the seven Lys residues was
MRAS, Myc-LZTR1 and HA-Ub (10 $\mu \mathrm{g}: 2 \mu \mathrm{g}: 7 \mu \mathrm{g}$ ratio of RAS: LZTR1:Ub). c In vivo ubiquitination assays were carried out as in (b) using HRAS-WT and HRAS-K170R mutant. d HEK293 cells were transfected with HRAS-WT, HRAS-G12V, HRAS-K170R and HRAS-G12V/K170R in the presence or absence of Myc-LZTR1. Forty-eight hours later, we evaluated the influence of LZTR1 on HRAS levels. In (d), cells were seeded in $60 \mathrm{~mm}$ dishes and transfected with transfected with HRAS and Myc-LZTR1 (2.5 $\mu \mathrm{g}: 0 \mu \mathrm{g}$ or $2.5 \mu \mathrm{g}: 2.5 \mu \mathrm{g}$ ratio of RAS:LZTR1)

mutated to Arg. The polyubiquitination levels of RAS decreased in HA-Ub-K48R- or HA-Ub-K63R-expressing cells (Fig. 5b). These results suggest that the degradative K48-linked chain is involved in LZTR1-mediated RAS polyubiquitination. The nondegradative K63-linked chain was also found to contribute to LZTR1-mediated RAS polyubiquitination, suggesting that LZTR1 has a distinct role, such as altering the cellular localization of RAS. Additionally, a decrease was observed in Ub-K33Rexpressing cells (Fig. 5b). Although little is known about Ub-K33-linked chains inducing the degradation of ubiquitinated proteins, our data suggest that Ub-K33-linked chains are involved in LZTR1-mediated RAS degradation.

\section{The association of LZTR1 and RAS-GTPases with autophagy}

The ability of conjugated polyubiquitin chains to confer selectivity to autophagy is becoming apparent [30, 31]. Nibe et al. reported that a Ub-K33-linked polyubiquitinated protein colocalized with autophagy-related proteins, including LC3B and its interacting protein SQSTM1/p62 [32]. In Supplementary Fig. S5a, we found that MRAS, NRAS, and KRAS also interacted with SQSTM1/p62 and 

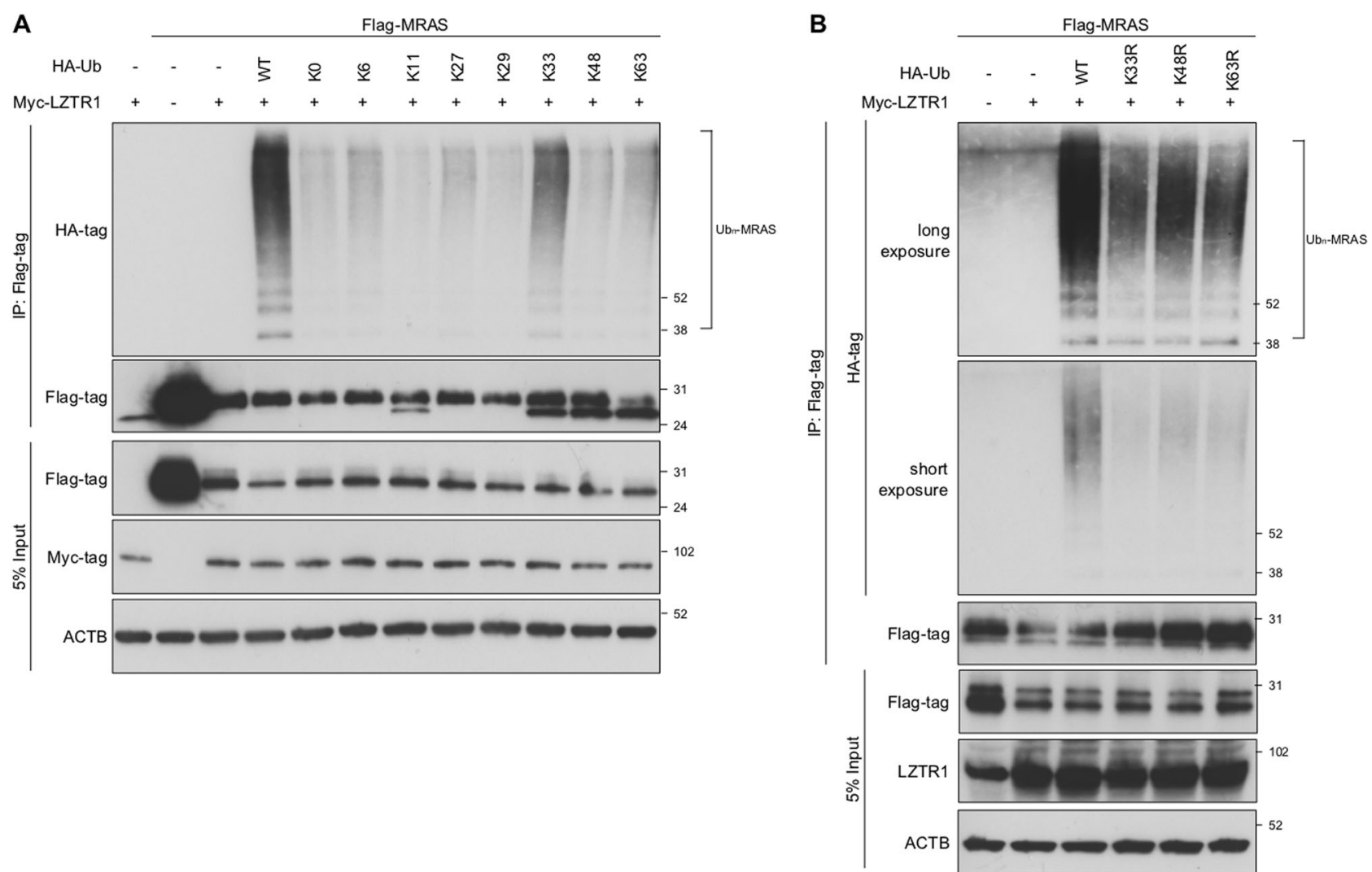

Fig. 5 Identification of the type of polyubiquitin chain involved in LZTR1-dependent RAS polyubiquitination. a, b In vivo ubiquitination assays were performed as shown in Fig. 2, and polyubiquitination

LC3B, whereas HRAS weakly or scarcely bound to SQSTM1/p62. Notably, Flag-RAS-GTPases interacted strongly with LC3B-II, the lipidated form of LC3B. Moreover, LZTR1 interacted with SQSTM1/p62 and LC3B-II (Supplementary Fig. S5b). Therefore, we hypothesized that RAS proteins are degraded not only by the proteasome, but also by the autophagy system.

We treated cells with chloroquine, an autophagy inhibitor, and evaluated whether the reduction in RAS by LZTR1 is recovered by inhibition of autophagy. When we treated cells with chloroquine for $4 \mathrm{~h}$, the level of lipidated form of LC3B (LC3B-II) increased compared with that in vehicle-treated cells (Fig. 6a). The LC3B-II level increased by LZTR1 upon RAS co-expression, whereas LZTR1 expression alone did not increase the LC3B-II level compared with that in mock-transduced cells (Fig. 6a and Supplementary Fig. S5c). The increase of LC3B by LZTR1 and RAS co-expression was observed in immunofluorescence analyses (Fig. 6b). However, the LZTR1dependent reduction in RAS-GTPases was not recovered by chloroquine treatment for $4 \mathrm{~h}$ (Fig. 6a). In addition, in cells treated with chloroquine for $48 \mathrm{~h}$, the levels of NRAS and KRAS was slightly rescued in the presence of LZTR1 (Supplementary Fig. S5c). These results suggest that the status was evaluated by western blotting using anti-HA-tag, anti-Flagtag, anti-Myc-tag, and anti-ßactin antibodies. In vivo ubiquitination assays were carried out as in Fig. 4b

contribution of autophagy to LZTR1-mediated RAS degradation is minimal.

\section{Discussion}

In this study, we demonstrated that LZTR1 regulates the degradation of endogenous RAS and various types of expressed RAS proteins, including MRAS, HRAS, NRAS, and KRAS, mainly via the ubiquitin-proteasome pathway. We first demonstrated that the expression of endogenous RAS increased in LZTRI-knockdown cells and decreased upon LZTR1 overexpression in a dose-dependent manner. By performing detailed analyses of the mechanism underlying the control of RAS expression by LZTR1, we determined that LZTR1 facilitates the polyubiquitination and degradation of RAS via the ubiquitin-proteasomal degradation pathway. Furthermore, our results suggest a possibility that LZTR1 and RAS are involved in autophagy through the interaction with SQSTM1/p62 and LC3B. The proposed model for LZTR1 function is shown in Fig. 7.

A growing body of evidence shows that the activities of RAS molecules are regulated by site-specific ubiquitination, in addition to GDP/GTP exchange. Mono- or 

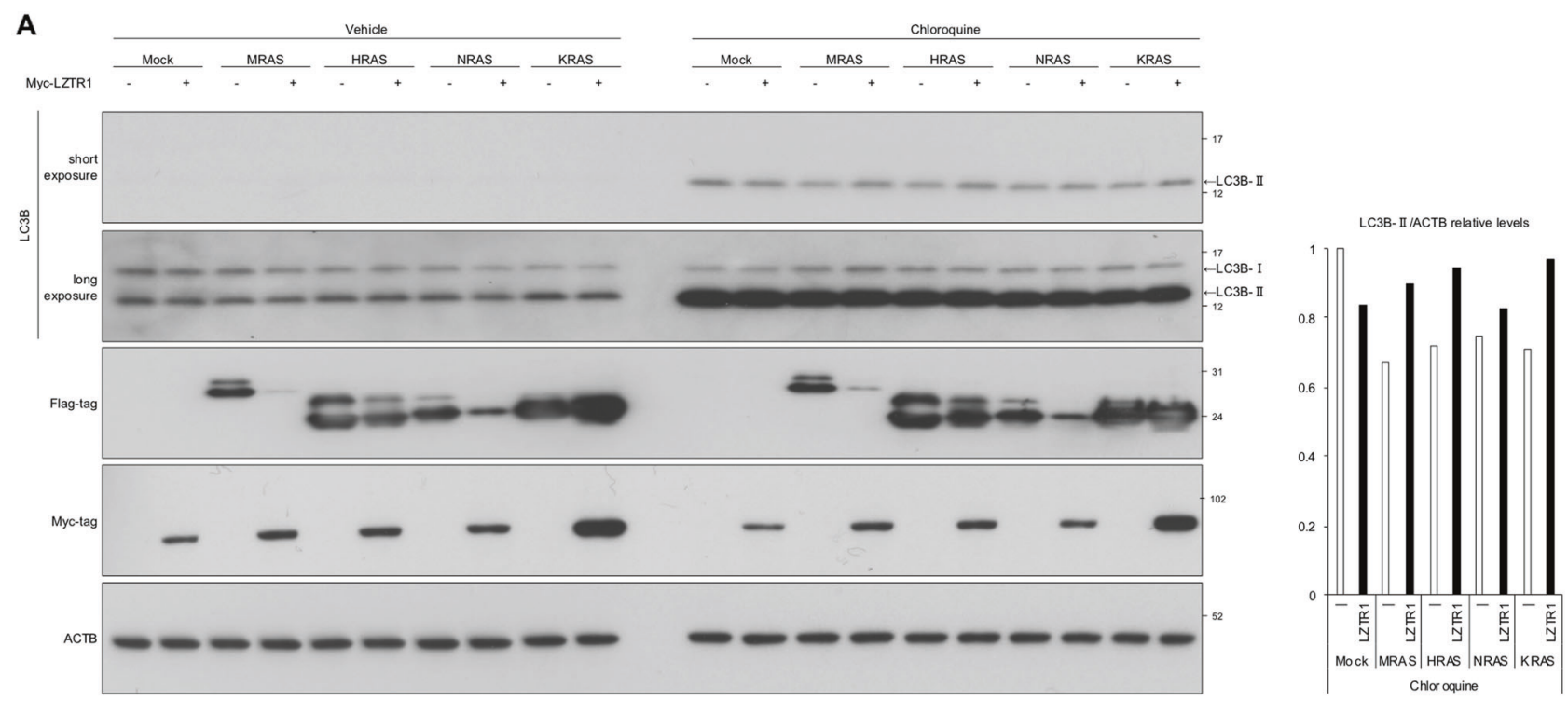

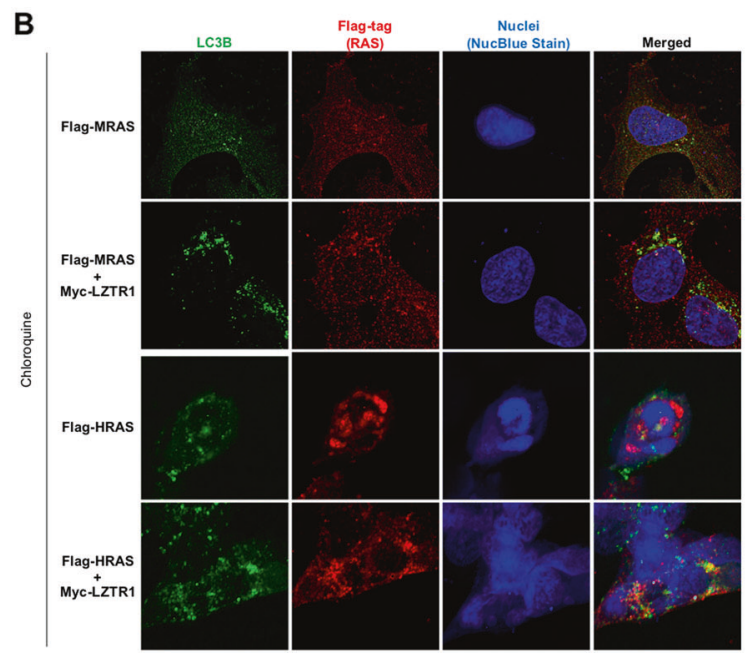

Fig. 6 LZTR1 and RAS might contribute to autophagy. a Cells were seeded in $60 \mathrm{~mm}$ dishes and transfected with transfected with RAS and Myc-LZTR1 (5:1 $\mathrm{g}$ ratio of RAS:LZTR1) for $24 \mathrm{~h}$, followed by treatment with $50 \mu \mathrm{M}$ chloroquine for $4 \mathrm{~h}$. We evaluated LC3B-II levels by western blot. Band densities were analyzed using Image J software as in Fig. 1d. b Under the same conditions as in B, the

di-ubiquitinated-RAS proteins show altered cellular localization and GTP loading. Consequently, such changes induce the activation or inactivation of RAS proteins, including HRAS and KRAS [33-36]. Of two research reports on LZTR1-dependent ubiquitination of RAS $[19,20]$, Steklov et al. suggested that (H)RAS-K170 is a critical target of LZTR1-dependent RAS ubiquitination and that its ubiquitination changes the cellular localization of RAS by attenuating the association of ubiquitinated-RAS with the membrane, which is non-degradative mechanism. Bigenzahn et al. also showed that inactivation of LZTR1 led to decreased ubiquitination and enhanced plasma membrane localization of endogenous KRAS. However, these reports

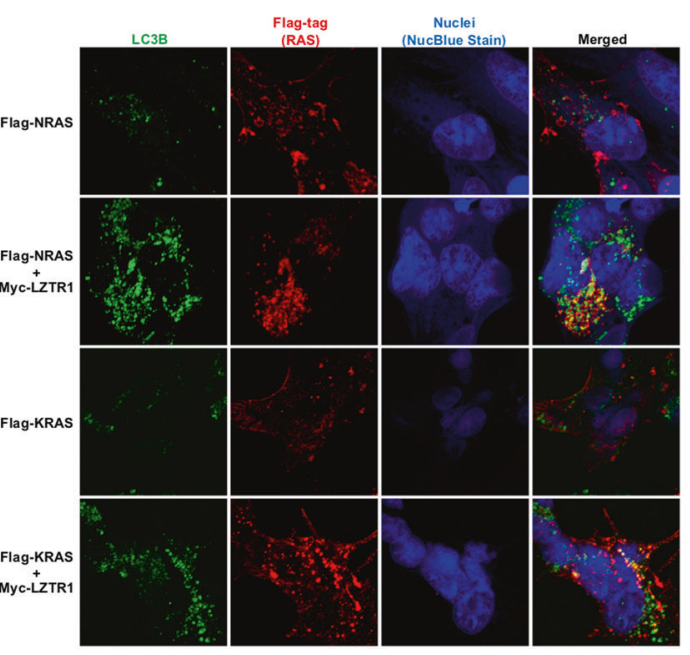

cellular colocalization of Flag-RAS and LC3B in HEK293 cells. We then stained the cells with anti-LC3B (green), anti-Flag-tag (red), and NucBlue Stain (nuclei, blue). The yellow arrows indicate representative locations where RAS and LC3B overlap. We used MRASpcDNA, HRAS-pcDNA, NRAS-pcDNA and KRAS-pCAGGS expression plasmids

did not describe the presence of an LZTR-mediated RAS degradation system. In the current study, we revealed that LZTR1 facilitates the polyubiquitination and degradation of endogenous RAS and various types of expressed RAS proteins, via the ubiquitin-proteasome pathway (Figs. 1 and 2). Co-immunoprecipitation assays showed that LZTR1 homodimerized with itself to form a heterotetrametric complex of two LZTR1, CUL3, and RAS (Fig. 3). Furthermore, this LZTR1-mediated RAS degradation induced the inactivation of MAPK signaling followed by the inhibition of cell growth (Figs. 1 and 2, and Supplementary Fig. S1). Regarding the degradation mechanism of the RAS family, Jeong et al. and Lee et al. showed that glycogen 

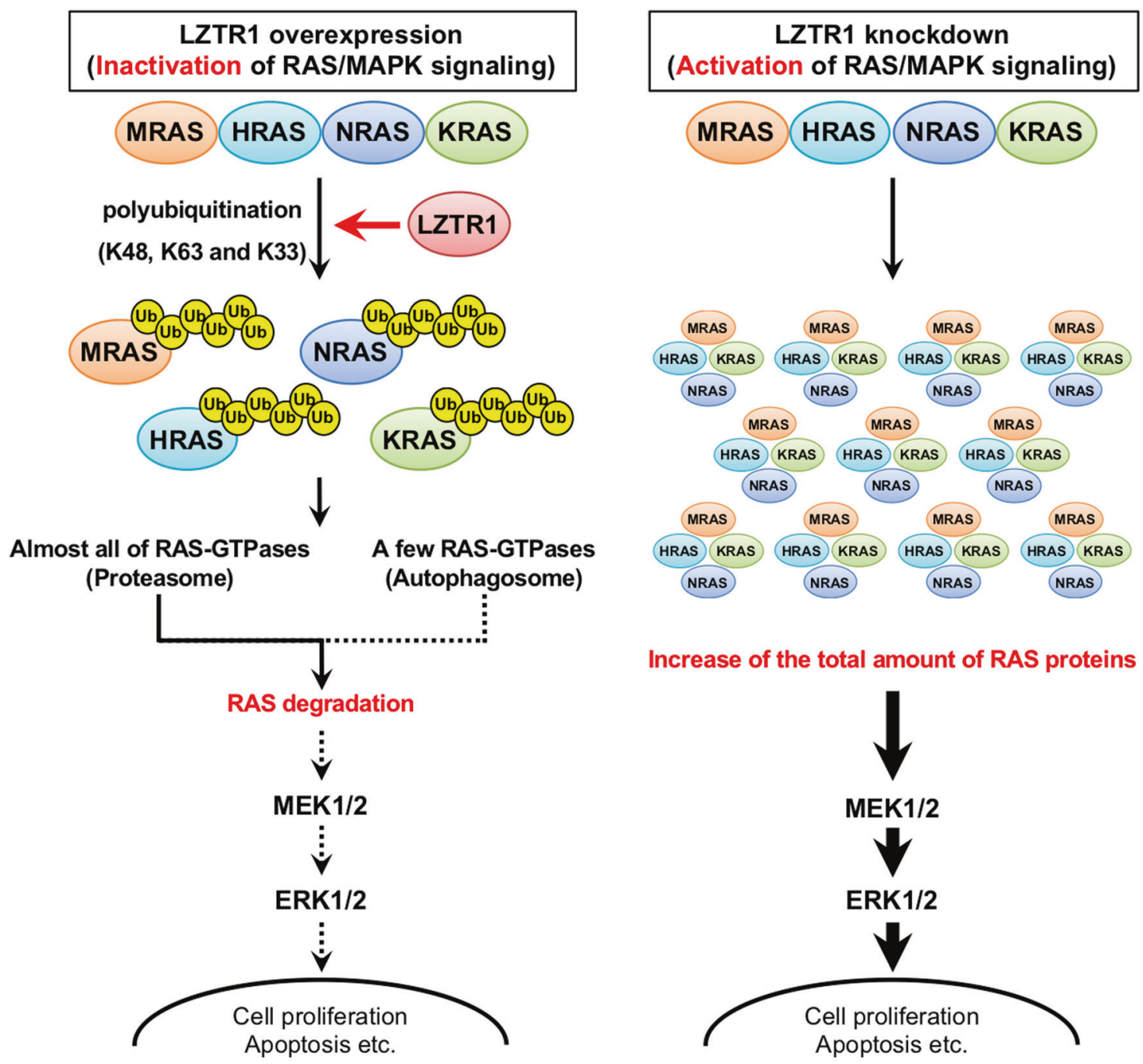

Fig. 7 The proposed model for LZTR1 function in the RAS/MAPK signaling pathway. RAS/MAPK signaling regulates cell survival and apoptosis. Our regulatory mechanism involves the RAS degradation via the proteasome and autophagy

synthase kinase $3 \beta$ (GSK3 $\beta$ ), a negative regulator of the $\mathrm{Wnt} / \beta$-catenin pathway, contributes to the phosphorylation of HRAS and the subsequent recruitment of $\beta$-TrCP-E3 ligase to phosphorylated-HRAS, leading to the degradation of HRAS [37, 38], but not other RAS molecules. To the best of our knowledge, our findings are the first to show that LZTR1 functions as a "multiple RAS killer protein" regardless of the type of RAS-GTPase.

Castel et al. recently reported that LZTR1 regulates polyubiquitination and degradation of RIT1 [39], supporting our finding that LZTR1 has a degradative effect on RAS-GTPases. RIT1 is a small GTPase, and mutations in RITl cause Noonan syndrome [14, 40]. Castel et al. showed that LZTR1 interacts with RIT1 and MRAS but not with other RAS-GTPases. In contrast, we exhibited the interactions of LZTR1 with four RAS-GTPases, including MRAS, HRAS, NRAS and KRAS (Supplementary Fig. S5a). The difference between the two studies remains unknown. It is possible that the affinity for RAS molecules is different, and the affinity of LZTR1 may be stronger for RIT1 and MRAS than other RAS-GTPases. The difference in affinity between each RAS molecule and LZTR1 will be important to develop RAS-targeted therapeutic agents focusing on the function of LZTR1.

In the analysis of target Lys residues ubiquitinated by LZTR1, MRAS-K127, which corresponds to K117 in HRAS, NRAS and KRAS, and HRAS-K170, which is located in HVR, were ubiquitinated by LZTR1. Attenuation of RAS degradation was not observed in cells expressing LZTR1 and MRAS-K127R or HRAS-K170R (Fig. 4 and Supplementary Fig. S4). Castel et al. showed that K135R and K187R double mutation abolished RIT1 degradation by LZTR1 [39]. RIT1-K135 coresponds to K117/K127 in four RAS-GTPases, while RIT1-K187 is located in HVR. The Lys residues around HVRs were not contained in the six common residues we selected, because the amino acid 
sequences in HVRs greatly differ between each of RAS proteins. Although the HVR homology is low between HRAS and RIT1, both Lys residues in HVRs were ubiquitinated by LZTR1. Therefore, it is probable that Lys residues in HVR of MRAS have a potential to be ubiquitinated by LZTR1, but its ubiquitination only may not be enough for RAS degradation similarly to HRAS and RIT1. Taken together, these results and previous reports suggest that multiple Lys residues ubiquitinated by LZTR1 is needed for RAS degradation and that single site ubiquitination will not be enough for it.

We found that Ub-K33-linked chains greatly contribute to LZTR1-mediated RAS polyubiquitination (Fig. 5), and LZTR1 and RAS co-expression slightly increase the expression levels of lipidated form of LC3B (Fig. 6b). In Supplementary Fig. S5a, b, both LZTR1 and RAS could interact with SQSTM1/p62 and LC3B alone. Bigenzahn et al. demonstrated that LZTR1 colocalizes with LC3B [20], supporting our results showing that LZTR1 is involved in autophagy. Thus, although the detailed function remains unclear, it is possible that LZTR1 and RAS contribute the autophagy via the Ub-K33-linked chain. Future studies will be necessary to elucidate the detailed role of the Ub-K33-linked chain in LZTR1-mediated autophagy.

In summary, LZTR1 facilitates the polyubiquitination and degradation of four RAS proteins via the proteasomal degradation systems, leading to the inhibition of the RAS/MAPK signaling activities. In addition, our results suggest a possible contribution of LZTR1 to autophagy. Since Motta et al. recently reported that pathogenic mutants of LZTR1 regulate the RAS/MAPK signaling pathway [41] as observed in our results, it is highly possible that dysfunction of the LZTR1-mediated RAS degradation system causes RASopathies. We believe that our present findings are fundamental for the exploration of the pathogenic mechanisms and establishing a common therapeutic strategy for RAS/MAPK signaling pathway-related diseases.

Acknowledgements We thank Shingo Takahara, Aya Shibui-Inoue, Yu Katata and Koki Nagai who contributed to the routine diagnostic work, and Yoko Tateda, Kumi Kato, and Riyo Takahashi for their technical assistance. We are grateful to J. Miyazaki, Osaka University, for supplying the pCAGGS expression vector. We also acknowledge the members of the Biomedical Research Core of Tohoku University School of Medicine and Biomedical Research Unit of Tohoku University Hospital for their support. This study was supported in part by the Grants-in-Aid by the Practical Research Project for Rare/Intractable Diseases from the Japan Agency for Medical Research and Development, AMED to Y.A. (18ek0109241h0002), and the Japan Society for the Promotion of Science (JSPS) KAKENHI Grant Number $17 \mathrm{H} 04223$ to YA and 18K15657 to TA.

Author contributions TA, IU, and SK performed the experiments and analyzed the data. TA and YA designed the project. TA wrote the manuscript, and SI, TN and YA revised the manuscript.

\section{Compliance with ethical standards}

Conflict of interest The authors declare that they have no conflict of interest.

Publisher's note: Springer Nature remains neutral with regard to jurisdictional claims in published maps and institutional affiliations.

\section{References}

1. Nacak TG, Leptien K, Fellner D, Augustin HG, Kroll J. The BTB-kelch protein LZTR-1 is a novel Golgi protein that is degraded upon induction of apoptosis. J Biol Chem. 2006;281: 5065-71.

2. Piotrowski A, Xie J, Liu YF, Poplawski AB, Gomes AR, Madanecki $P$, et al. Germline loss-of-function mutations in LZTR1 predispose to an inherited disorder of multiple schwannomas. Nat Genet. 2014;46:182.

3. Furukawa M, He YJ, Borchers C, Xiong Y. Targeting of protein ubiquitination by BTB-Cullin 3-Roc1 ubiquitin ligases. Nat Cell Biol. 2003;5:1001-7.

4. Geyer R, Wee S, Anderson S, Yates J, Wolf DA. BTB/POZ domain proteins are putative substrate adaptors for cullin 3 ubiquitin ligases. Mol Cell. 2003;12:783-90.

5. Stogios PJ, Prive GG. The BACK domain in BTB-kelch proteins. Trends Biochem Sci. 2004;29:634-7.

6. Lu A, Pfeffer SR. A CULLINary ride across the secretory pathway: more than just secretion. Trends Cell Biol. 2014; 24:389-99.

7. Frattini V, Trifonov V, Chan JM, Castano A, Lia M, Abate F, et al. The integrated landscape of driver genomic alterations in glioblastoma. Nat Genet. 2013;45:1141.

8. Franceschi S, Lessi F, Aretini P, Mazzanti CM, Menicagli M, La Ferla M, et al. Molecular portrait of a rare case of metastatic glioblastoma: somatic and germline mutations using whole-exome sequencing. Neuro-Oncol. 2016;18:298.

9. Yamamoto GL, Aguena M, Gos M, Hung C, Pilch J, Fahiminiya $\mathrm{S}$, et al. Rare variants in SOS2 and LZTR1 are associated with Noonan syndrome. J Med Genet. 2015;52:413-21.

10. Johnston JJ, van der Smagt JJ, Rosenfeld JA, Pagnamenta AT, Alswaid A, Baker EH, et al. Autosomal recessive Noonan syndrome associated with biallelic LZTR1 variants. Genet Med. 2018;20:1175-85.

11. Romano AA, Allanson JE, Dahlgren J, Gelb BD, Hall B, Pierpont $\mathrm{ME}$, et al. Noonan syndrome: clinical features, diagnosis, and management guidelines. Pediatrics. 2010;126:746-59.

12. Tidyman WE, Rauen KA. The RASopathies: developmental syndromes of Ras/MAPK pathway dysregulation. Curr Opin Genet Dev. 2009;19:230-6.

13. Aoki Y, Niihori T, Inoue S-I, Matsubara Y. Recent advances in RASopathies. J Hum Genet. 2016;61:33.

14. Aoki Y, Niihori T, Banjo T, Okamoto N, Mizuno S, Kurosawa K, et al. Gain-of-function mutations in RIT1 cause Noonan syndrome, a RAS/MAPK pathway syndrome. Am J Hum Genet. 2013;93:173-80.

15. Vissers LE, Bonetti M, Paardekooper Overman J, Nillesen WM, Frints SG, de Ligt $\mathrm{J}$, et al. Heterozygous germline mutations in A2ML1 are associated with a disorder clinically related to Noonan syndrome. Eur J Hum Genet. 2015;23:317-24.

16. Gripp KW, Aldinger KA, Bennett JT, Baker L, Tusi J, PowellHamilton N, et al. A novel rasopathy caused by recurrent de novo missense mutations in PPP1CB closely resembles Noonan syndrome with loose anagen hair. Am J Med Genet A. 2016;170: 2237-47. 
17. Higgins EM, Bos JM, Mason-Suares H, Tester DJ, Ackerman JP, MacRae CA, et al. Elucidation of MRAS-mediated Noonan syndrome with cardiac hypertrophy. JCI Insight. 2017;2:e91225.

18. Umeki I, Niihori T, Abe T, Kanno S-i, Okamoto N, Mizuno S, et al. Delineation of LZTR1 mutation-positive patients with Noonan syndrome and identification of LZTR1 binding to RAF1-PPP1CB complexes. Hum Genet. 2018;138:1-15.

19. Steklov M, Pandolfi S, Baietti M, Batiuk A, Carai P, Najm P, et al. Mutations in LZTR1 drive human disease by dysregulating RAS ubiquitination. Science. 2018;362:eaap7607.

20. Bigenzahn JW, Collu GM, Kartnig F, Pieraks M, Vladimer GI, Heinz LX, et al. LZTR1 is a regulator of RAS ubiquitination and signaling. Science. 2018;362:eaap8210.

21. Niihori T, Aoki Y, Narumi Y, Neri G, Cavé H, Verloes A, et al. Germline KRAS and BRAF mutations in cardio-facio-cutaneous syndrome. Nat Genet. 2006;38:294.

22. Kobayashi A, Kang M-I, Okawa H, Ohtsuji M, Zenke Y, Chiba T, et al. Oxidative stress sensor Keap1 functions as an adaptor for Cul3-based E3 ligase to regulate proteasomal degradation of Nrf2. Mol Cell Biol. 2004;24:7130-9.

23. Huttlin EL, Ting L, Bruckner RJ, Gebreab F, Gygi MP, Szpyt J, et al. The BioPlex network: a systematic exploration of the human interactome. Cell. 2015;162:425-40.

24. Bardwell VJ, Treisman R. The POZ domain: a conserved proteinprotein interaction motif. Genes Dev. 1994;8:1664-77.

25. Zhuang M, Calabrese MF, Liu J, Waddell MB, Nourse A, Hammel M, et al. Structures of SPOP-substrate complexes: insights into molecular architectures of BTB-Cul3 ubiquitin ligases. Mol Cell. 2009;36:39-50.

26. Mena EL, Kjolby RA, Saxton RA, Werner A, Lew BG, Boyle JM, et al. Dimerization quality control ensures neuronal development and survival. Science. 2018;362:eaap8236.

27. Komander D, Rape M. The ubiquitin code. Annu Rev Biochem. 2012;81:203-29.

28. Huang X, Dixit VM. Drugging the undruggables: exploring the ubiquitin system for drug development. Cell Res. 2016;26:484.

29. Weissman AM. Ubiquitin and proteasomes: themes and variations on ubiquitylation. Nat Rev Mol Cell Biol. 2001;2:169.

30. Delgado ME, Dyck L, Laussmann MA, Rehm M. Modulation of apoptosis sensitivity through the interplay with autophagic and proteasomal degradation pathways. Cell Death Dis. 2015;5:e1011.
31. Shaid S, Brandts C, Serve H, Dikic I. Ubiquitination and selective autophagy. Cell Death Differ. 2013;20:21.

32. Nibe Y, Oshima S, Kobayashi M, Maeyashiki C, Matsuzawa Y, Otsubo K, et al. Novel polyubiquitin imaging system, PolyUb-FC, reveals that K33-linked polyubiquitin is recruited by SQSTM1/ p62. Autophagy. 2018;14:347-58.

33. Jura N, Scotto-Lavino E, Sobczyk A, Bar-Sagi D. Differential modification of Ras proteins by ubiquitination. Mol Cell. 2006;21: 679-87.

34. Baker R, Lewis SM, Sasaki AT, Wilkerson EM, Locasale JW, Cantley LC, et al. Site-specific monoubiquitination activates Ras by impeding GTPase-activating protein function. Nat Struct Mol Biol. 2013;20:46-52.

35. Baker R, Wilkerson EM, Sumita K, Isom DG, Sasaki AT, Dohlman HG, et al. Differences in the regulation of K-Ras and HRas isoforms by monoubiquitination. J Biol Chem. 2013;288: 36856-62.

36. Sasaki AT, Carracedo A, Locasale JW, Anastasiou D, Takeuchi $\mathrm{K}$, Kahoud ER. et al. Ubiquitination of K-Ras enhances activation and facilitates binding to select downstream effectors. Sci Signal. 2011;4:ra13.

37. Jeong W-J, Yoon J, Park J-C, Lee S-H, Lee S-H, Kaduwal S. et al. Ras stabilization through aberrant activation of Wnt/B-catenin signaling promotes intestinal tumorigenesis. Sci Signal. 2012;5: ra30.

38. Lee SK, Jeong WJ, Cho YH, Cha PH, Yoon JS, Ro EJ, et al. betaCatenin-RAS interaction serves as a molecular switch for RAS degradation via GSK3beta. EMBO Rep. 2018;19:e46060.

39. Castel P, Cheng A, Cuevas-Navarro A, Everman DB, Papageorge AG, Simanshu DK, et al. RIT1 oncoproteins escape LZTR1mediated proteolysis. Science. 2019;363:1226-30.

40. Takahara S, Inoue S-i, Miyagawa-Tomita S, Matsuura K, Nakashima Y, Niihori T, et al. New Noonan syndrome model mice with RIT1 mutation exhibit cardiac hypertrophy and susceptibility to $\beta$-adrenergic stimulation-induced cardiac fibrosis. EBioMedicine. 2019;42:43-53.

41. Motta M, Fidan M, Bellacchio E, Pantaleoni F, Schneider-Heieck $\mathrm{K}$, Coppola S, et al. Dominant Noonan syndrome-causing LZTR1 mutations specifically affect the kelch domain substraterecognition surface and enhance RAS-MAPK signaling. Hum Mol Genet. 2018;28:1007-22. 